\title{
TESTE EMPÍRICO DA EFICIÊNCIA DO MERCADO BRASILEIRO NA OCORRÊNCIA DE EVENTOS FAVORÁVEIS E DESFAVORÁVEIS
}

\author{
Glauber de Castro Barbosa ${ }^{1}$ \\ Otávio Ribeiro de Medeiros ${ }^{2}$
}

\begin{abstract}
Resumo: Este artigo tem o objetivo de analisar o comportamento do mercado acionário brasileiro entre os anos de 2001 e 2005 para verificar a existência de eficiência de mercado após a ocorrência de eventos (choques) favoráveis e desfavoráveis. Para isso realizou-se um estudo de evento, no qual o retorno do Ibovespa - índice do mercado brasileiro - foi regredido contra o retorno do índice Dow Jones, representativo da bolsa de Nova lorque, adotado como "proxy" para o índice do mercado mundial. Delimitou-se um desvio de $+2,5 \%(-2,5 \%)$ nos resíduos da regressão para determinar eventos positivos (negativos) e calculou-se o Retorno Anormal Acumulado para um período de dez dias após o evento. Constatou-se que tanto depois de choques positivos quanto de negativos a eficiência do mercado não foi observada, mas sim uma reposta exagerada (overreaction) aos choques.
\end{abstract}

Palavras-chave: Choques econômicos. Eficiência de mercado. Overreaction. Uncertain information hypothesis. Underreaction. Retorno anormal acumulado (RAA).

\section{INTRODUÇÃO}

O mundo das finanças vem sofrendo transformações ao longo do tempo. Modelos são criados, alguns são refutados e outros são adaptados. A hipótese de mercado eficiente (HME) sempre dominou as finanças modernas, mas desde que Daniel Khaneman e Amos Tversky 3 começaram suas pesquisas com 0 intuito de demonstrar que o investidor não é tão racional como a HME previa vários testes empíricos foram realizados para tentar invalidar ou adaptar essa hipótese.

Este artigo aborda um estudo do comportamento do mercado acionário brasileiro. A intenção é verificar a existência de eficiência nesse meio, pois o mercado nacional, por não ser, ainda, tão desenvolvido, leva à possibilidade de ineficiência e, além disso, não desperta tanto interesse em âmbito internacional. Entretanto, pelo fato de o Brasil ser um país emergente, e, tendo em vista a globalização, essa situação tende a mudar. Neste estudo, utilizam-se índices de mercado como "benchmarks" a fim eliminar as anomalias específicas do mercado e examinar seu comportamento. A finalidade principal do estudo foi testar, por meio de um estudo de evento, as seguintes hipóteses:

- $\mathrm{H}_{1}$ : o mercado brasileiro no período estudado é eficiente após eventos favoráveis.

- $\mathrm{H}_{2}$ : o mercado brasileiro no período estudado é eficiente após eventos desfavoráveis.

\footnotetext{
1 Especialista em Gestão Pública pela Fortium, Sobradinho, DF. E-mail: glaubercb@gmail.com.

2 Departamento de Ciências Contábeis e Atuariais, UnB, BRASILIA, DF. E-mail: otavio@ unb.br.
} 
A análise segue os efeitos nos retornos dos índices depois de notícias favoráveis e desfavoráveis. 0 benchmark adotado para gerar retornos anormais do mercado brasileiro é o índice Dow Jones, que se refere à Bolsa de Valores de Nova lorque (NYSE). O retorno do índice de mercado brasileiro, Ibovespa, foi regredido em relação ao retorno do índice Dow Jones para gerar os eventos.

Para efeito de análise, serão apresentadas, também, as seguintes hipóteses: a Uncertain Information Hypothesis $(\mathrm{UIH})$, a Hipótese do Overreaction $(\mathrm{HO})$ e a Hipótese do Underreaction (HU).

A pesquisa foi limitada entre janeiro de 2001 e dezembro de 2005, e sua bibliografia básica encontra-se toda em inglês, uma vez que ainda não existem muitas pesquisas nessa área no Brasil.

$O$ trabalho encontra-se dividido em cinco seções, sendo que: a seção 1 contém a introdução e os objetivos; a 2 discorre sobre a revisão de literatura apresentando algumas evidências empíricas; a 3 apresenta os dados e descreve a metodologia do estudo; a seção 4 exibe a análise dos resultados e a seção 5 relata a conclusão.

\section{REVISÃO DE LITERATURA}

Utilizou-se a metodologia de estudo de evento para analisar as reações do mercado de capitais brasileiro a eventos ou choques positivos e negativos. Entende-se por choque econômico o evento exógeno que traz impacto à economia ou aos mercados de um país. Como exemplos de choque citamse: o choque do petróleo em 1973, o atentado terrorista de 11 de setembro nos Estados Unidos e a invasão do Iraque.

Na Figura 1, estão representadas graficamente as reações previstas na $\mathrm{HME}, \mathrm{UIH}, \mathrm{HO}$ e $\mathrm{HU}$ no que diz respeito aos eventos favoráveis (positivos) e desfavoráveis (negativos). Desde quando foi proposta pela primeira vez por Fama (1970) a hipótese de mercado eficiente (HME) vem sendo testada empiricamente. Existem três condições que precisam ser alcançadas para se chegar a HME: primeiramente não deve haver custos de transação na negociação dos títulos; em segundo lugar, todas as informações devem estar disponíveis para todos os participantes do mercado, sem qualquer custo; e, por fim, todos devem ter a mesma percepção da implicação da informação para o preço corrente e para a distribuição de probabilidades de preços futuros.

Essa hipótese tem como principais pressupostos: que o mercado tem de ser competitivo, ou seja, o preço dos títulos pode flutuar de forma a atingir um equilíbrio que seria a igualdade entre a quantidade ofertada e a demandada; um investidor não pode ter sistematicamente um retorno que, ajustado pelo risco, seja maior que o retorno do mercado; toda informação nova deve ser refletida no preço do título, imediatamente ou em um período curto de tempo, o que remete ao conceito de racionalidade do investidor. Segundo Fama (1970) ao presumir que os investidores sejam racionais a hipótese de mercado eficiente pressupõe que os preços de todos os ativos refletem exatamente o valor presente do fluxo de caixa futuro gerado para cada ativo. Por conseqüência, os investidores processam as novas informações imediatamente após o recebimento dela, elevando (reduzindo) o preço de determinado ativo sempre que a notícia implicar aumento (redução) do fluxo de caixa futuro, sendo que esse aumento (redução) do preço corresponderá exatamente à variação do valor presente do fluxo de caixa futuro. Da mesma forma, se não houver notícia, não haverá alteração no preço de um ativo. 


\section{HIPÓTESE DE MERCADO EFICIENTE}

Eventos Favoráveis

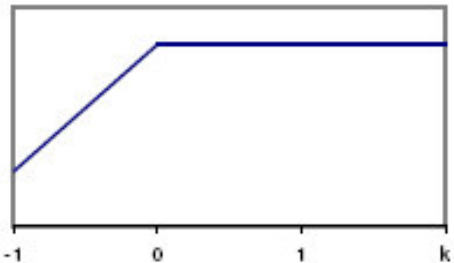

Eventos Desfavoráveis

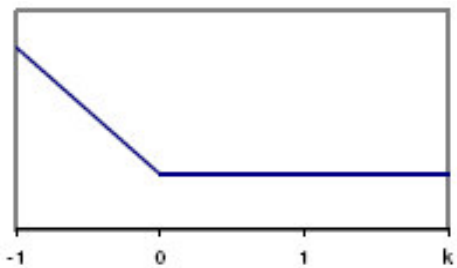

\section{UNCERTAIN INFORMATION HYPOTHESIS}

Eventos Favoráveis

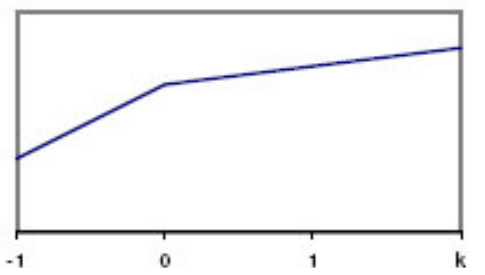

HIPÓTESE DE OVERREACTION

Eventos Favoráveis

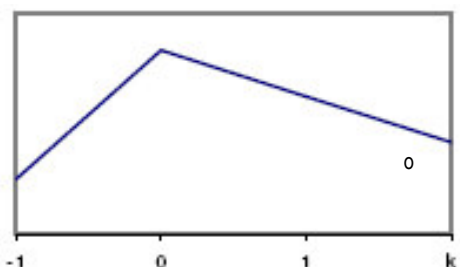

Eventos Desfavoráveis

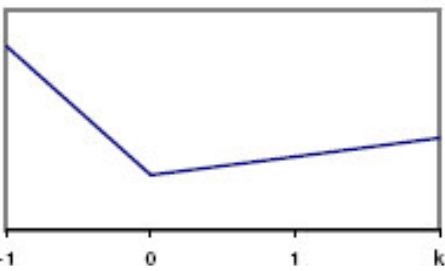

Eventos Desfavoráveis

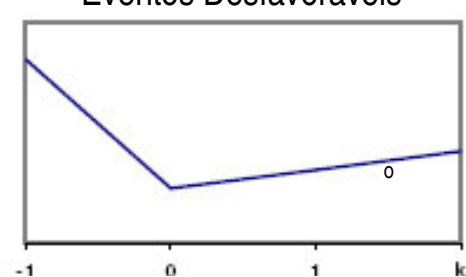

HIPÓTESE DE UNDERREACTION

Eventos Favoráveis



Eventos Desfavoráveis

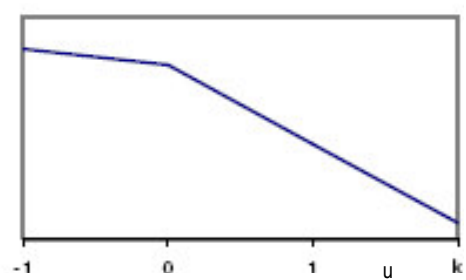

$\mathrm{t}=0$ = data do evento

$\mathrm{p}=$ preço inicial

$p^{e}=$ preço de equilíbrio

$\mathrm{p}^{0}=$ preço na hipótese de overreaction

$\mathrm{p}^{\mathrm{ui}}=$ preço na hipótese de UIH

$\mathrm{Pu}^{\mathrm{u}}=$ preço na hipótese de underreaction

Figura 1 - Reações previstas na HME, UIH, HO e HU

Fonte: Adaptado de Medeiros (2006) 
Algumas informações podem afetar os preços dos títulos mais rapidamente do que outras e isso pode ocorrer de três formas distintas, de acordo com o quadro abaixo:

\begin{tabular}{|l|l|}
\hline Fraca & As informações contidas nos preços passados não são relevantes para a obtenção de retornos extraordinários. \\
\hline Semiforte & Os investidores não podem obter retornos extraordinários baseados em informações públicas. \\
\hline Forte & $\begin{array}{l}\text { Os investidores não podem obter retornos extraordinários usando quaisquer informações, públicas ou } \\
\text { privilegiadas (insider information). }\end{array}$ \\
\hline
\end{tabular}

\section{Quadro 1: Formas da HME.}

A Uncertain Information Hypothesis (UIH) que traduzida para o português seria a hipótese da informação em condições de incerteza propõe-se a descrever o comportamento racional dos agentes em um ambiente de incerteza. De acordo com Shachmurove (2002) a teoria prediz que a volatilidade dos retornos aumentará depois de um anúncio. Especificamente depois de anúncios negativos a volatilidade é maior do que nos positivos. Isto é uma conseqüência racional dos agentes que têm aversão ao risco. Brown, Harlow e Tiniç (1988) propuseram inicialmente a UIH com a finalidade de fornecer uma versão mais flexível e mais realista da HME, baseado em regras completamente racionais para decisão do investidor.

Os autores apresentam os investidores como: (I) racionais no sentido de von NeumannMorgenstern (isto é, eles maximizam a utilidade prevista) e dão forma a expectativas racionais; (II) são avessos ao risco; (III) o mercado incorpora rapidamente toda a informação disponível aos preços das ações; e (IV) as principais surpresas podem ser identificadas como notícias boas ou más, todavia, a extensão de seu impacto em preços de mercado é incerta. Entretanto, os investidores podem dar forma à distribuição condicional das probabilidades de retorno, dadas notícias boas ou más. A UIH prediz então que, no momento após a nova informação, o risco e o retorno previstos das empresas afetadas aumentam de maneira sistemática.

A Hipótese do Overreaction $(\mathrm{HO})$ que também poderia se chamar sobre-reação ou reação exagerada decorre da heurística da "representatividade", de autoria de Tversky e Kahneman (1974 apud SOARES e SERRA, 2005) na qual os investidores tendem a sobrevalorizar a informação recente, negligenciando ou dando menos importância à informação passada na sua revisão de expectativas, com base nos seus julgamentos subjetivos de probabilidade. Os preços refletiriam em excesso essa chegada da nova informação sendo reconduzidos a valores de equilíbrio, no médio e longo prazo. Dessa forma, eventos favoráveis influenciam o mercado a estabelecer preços acima da taxa média de retorno. Entretanto, em algum instante, o mercado percebe que os preços foram ajustados demasiadamente altos, reajustando-os a valores mais realísticos. Por sua vez, quando ocorrem eventos desfavoráveis, o mercado reduz demasiadamente os preços e, com o passar do tempo, estes tendem para valores mais coerentes.

A Hipótese do Underreaction (HU) baseia-se no viés do "conservadorismo", proposto por Edwards (1968 apud SOARES e SERRA, 2005) por meio do qual os investidores se ajustam lentamente à chegada de nova informação, incorporando de forma gradual as suas expectativas ao sistema de preços, motivando o efeito de transição dos retornos. Sendo assim, quando ocorrem eventos favoráveis o mercado estabelece preço abaixo do ideal e, com o passar do tempo, ele vai aumentando até chegar a um patamar considerado ideal e, quando acontecem eventos desfavoráveis, o mercado é conservador e não baixa tanto o preço e, à medida que o tempo passa, esse preço 
continua baixando até alcançar uma cotação mais realista.

Existem algumas evidências empíricas quanto à validade ou não das hipóteses de mercado apresentadas. Brown, Harlow e Tiniç (1988) utilizaram o índice CRSP4 e as 200 maiores ações listadas no S\&P 500 para testar a UIH em um período que vai de julho de 1962 a dezembro de 1985. Constataram que os retornos médios após a divulgação, que seguem eventos favoráveis e desfavoráveis, tendem a ser significativamente positivos. Entretanto, observaram que as correlações entre as mudanças imediatas do preço causadas pelos eventos e 0 sentido das mudanças subseqüentes do preço são extremamente baixas. Os autores discutem que esses resultados são consistentes com a UIH, pois as respostas que seguem aos eventos individuais são aleatórias.

Shachmurove (2002) estudou o comportamento de treze mercados de pequenos países europeus para encontrar sustentação para a presença da HME e da UIH nesses mercados. Os treze países objeto foram: Bélgica, Dinamarca, Finlândia, Grécia, Irlanda, Luxemburgo e os Países Baixos Noruega, Portugal, Espanha, Suécia, Suíça e Turquia. Nesse estudo ficou evidenciado que os investidores desses países geralmente reagem à informação incerta com comportamento eficiente e racional. $O$ autor observou, também, que inicialmente os investidores ajustavam os preços abaixo de seu valor de mercado. Afirma que o resultado dos testes-padrão de passeio aleatório são evidentes. Conseqüentemente, acredita que, aderindo ao paradigma do UIH e do passeio aleatório (randon walk) típico da HME, a maioria dos mercados examinados é eficiente.

Medeiros (2006) analisou o comportamento do mercado acionário brasileiro no período de abril de 1994 a outubro de 2005 com a utilização do lbovespa e do índice Dow Jones como uma proxy do mercado mundial. Encontrou evidências de que o mercado brasileiro assumiu, no período estudado, a $\mathrm{OH}$ para eventos positivos (favoráveis) e a UH para eventos negativos (desfavoráveis). Uma resposta de aumento da volatilidade aos eventos positivos e negativos refutou a HME e, adicionalmente, observou-se, após a divulgação, propensão para que as variações positivas fossem maiores do que as negativas contradizendo assim a UIH.

Soares e Serra (2005) em seu estudo descreveram e testaram a existência de Overreaction e Undereaction por parte dos investidores no mercado de ações português. 0 objetivo era investigar, especificamente, a existência desses fenômenos no mercado português. Os resultados encontrados indicam a existência de ambos os fenômenos.

\section{DADOS E METODOLOGIA}

\subsection{DADOS}

Durante a pesquisa foram coletados os índices: Ibovespa e Dow Jones, com o objetivo de testar a eficiência dez dias depois de um desvio inesperado no índice brasileiro relativo ao índice Dow Jones. Utilizou-se 0 índice da bolsa de Nova lorque, uma vez que a Bolsa Brasileira acompanha 0 movimento do mercado norte-americano, e o mesmo pode ser uma representação do mercado mundial por ser o mais forte e desenvolvido do mundo.

O lbovespa é o principal indicador médio do comportamento das principais ações negociadas na Bolsa de Valores de São Paulo - BOVESPA. As ações integrantes do índice respondem por mais de $80 \%$ do número de negócios e do volume financeiro verificados no mercado a vista da bolsa. 0 índice é calculado em tempo real, considerando os preços dos últimos negócios efetuados no mercado com ações componentes de sua carteira. Sendo o somatório dos pesos (quantidade teórica da ação

\footnotetext{
4 Índice do mercado norte-americano, divulgado pelo Center for Research in Security Prices - ligado a Universidade de Chicago.
} 
multiplicada pelo último preço dela) das ações integrantes de sua carteira teórica. A cada quadrimestre a carteira do Ibovespa é reavaliada, sua vigência é de quatro meses, vigorando para os períodos de janeiro a abril, maio a agosto e setembro a dezembro. A divulgação é feita pela rede de difusão da bolsa e também retransmitida por uma série de corretoras tendo a possibilidade de acompanhar on line seu comportamento. Atualmente, a BOVESPA é o maior centro de negociação com ações da América Latina, destaque que alcançou com um acordo histórico para a integração de todas as bolsas brasileiras em um único mercado de valores.

O Dow Jones é o índice utilizado para acompanhar a evolução dos negócios na New York Stock Exchange (NYSE) a Bolsa de Valores de Nova lorque. $O$ cálculo é feito com base na média das cotações entre as 30 empresas de maior importância na bolsa de valores, as 20 companhias ferroviárias mais destacadas e as 15 maiores empresas concessionárias de serviços públicos. A maior e mais importante bolsa de valores do mundo (NYSE) também conhecida como "Big Board", é composta de um conselho de 20 membros que acompanham e regulam as atividades comerciais de mais de 3000 empresas norte-americanas e estrangeiras.

Os índices foram coletados no sítio do Ipea (www.ipeadata.gov.br) com dados diários e a valores de fechamento. $O$ período de abrangência foi de janeiro de 2001 a dezembro de 2005 . Foram excluídos os dias sem negociação como feriados e finais de semana, bem como dias em que havia movimentação em uma bolsa, mas não tinha na outra. Por exemplo, no dia 4 de julho há movimento na BOVESPA, mas não na NYSE. A amostra foi composta de 1198 observações (dias).

\subsection{METODOLOGIA}

A metodologia utilizada na pesquisa foi a de estudo de eventos que, segundo Lamounier e Nogueira (2005) visa, a partir da hipótese de que os mercados sejam eficientes, mensurar o impacto da divulgação de uma dada informação no valor da empresa, mais especificamente, no comportamento dinâmico dos preços e dos retornos dessa firma nos mercados financeiros.

Seguindo a metodologia utilizada por Brown, Harlow e Tiniç (1988), Shachmurove (2002) e Medeiros (2006), a taxa de retorno do lbovespa foi regredida contra o retorno do Dow Jones, sendo 0 retorno do índice brasileiro a variável dependente e o retorno do norte-americano a variável independente. Para o presente estudo foram considerados eventos positivos (favoráveis) aqueles que tiveram os resíduos da regressão com desvio superior a $+2,5 \%$, e eventos negativos (desfavoráveis) os que tiveram os resíduos da regressão com desvio inferior a $-2,5 \%$, a regressão foi representada pela seguinte fórmula:

$$
R_{t}=\alpha+\beta R_{t}^{D J}+u_{t}(1.1)
$$

Em que $R_{t}$ é o retorno observado do índice brasileiro; $R_{t}^{D J}$ é o retorno observado do Dow Jones; $\alpha$ e $\beta$ são os coeficientes da regressão; e o u é o erro. Os retornos foram calculados pela forma logarítmica que implica uma distribuição de freqüência simétrica:

$$
R_{t}=\log \frac{I_{t}}{I_{t-1}} \quad \text { e } \quad R_{t}^{D J}=\log \frac{I_{t}^{D J}}{I_{t-1}^{D J}}
$$


Em que "log" é o operador logarítmico neperiano (natural); e $I_{t}$ e $I_{t}^{D J}$ são respectivamente 0 índice Ibovespa e o Dow Jones. O retorno esperado $\left(\hat{R}_{t}\right)$ do índice brasileiro é dado por:

$$
\hat{R}_{t}=\hat{\alpha}+\hat{\beta} R_{t}^{D J}
$$

Em que $\hat{\alpha}$ e $\hat{\beta}$ são os parâmetros da regressão estimados por mínimos quadrados ordinários (OLS). Os resíduos da regressão de (1.1), $\hat{u}_{t}$ são expressos por:

$$
\hat{u}_{t}=R_{t}-\hat{R}_{t}
$$

Um determinado resíduo é identificado como evento positivo (negativo) se for maior (menor) que $+2,5 \%(-2,5 \%)$ em relação à reta de regressão, isto é, por definição, um evento positivo ocorre no tempo $t$ se:

$$
\hat{u}_{t}>0,025,(1.5)
$$

enquanto que um evento negativo ocorre no tempo $t$ se:

$$
\hat{u}_{t}<-0,025(1.6)
$$

A data em que o evento ocorre é considerada a data zero, a data -1 é o dia anterior e observações relevantes são obtidas examinando os dez dias subseqüentes que vão da data +1 até +10. Para encontrar o retorno anormal $\left(R A_{t}\right)$ foram consideradas as médias dos resíduos $\left(\hat{u}_{t}\right)$ pelo número de eventos $d$ na data $t$ :

$$
R A_{t}=\frac{1}{n} \sum_{d=1}^{n} \hat{u}_{t} \quad, t=-1,0,+1, \ldots,+10
$$

Sendo $d=1, \ldots, n$, denota 0 número de eventos favoráveis ou desfavoráveis. Seguindo a metodologia, o retorno anormal acumulado $\left(R A A_{t}\right)$ na data té dado por:

$$
R A A_{t}=R A A_{t-1}+R A_{t}
$$

Em que $R A A_{t-1}$ é o retorno anormal acumulado na data $t-1$. 


\section{ANÁLISE DOS RESULTADOS}

$\mathrm{Na}$ Tabela 1 apresentam-se os valores do retorno anormal (RA) e do retorno anormal acumulado (RAA) das datas -1 até +10 após eventos (choques) positivos e negativos. Na amostra, foram encontrados 70 eventos favoráveis (positivos) e 78 desfavoráveis (negativos).

Tabela 1 - Reação aos choques positivos e negativos

\begin{tabular}{c|c|c|c|c}
\hline & \multicolumn{2}{|c|}{ CHOQUES POSITIVOS } & \multicolumn{2}{c}{ CHOQUES NEGATIVOS } \\
\hline Data & RA & RAA & RA & RAA \\
\hline-1 & $-0,003078$ & $-0,003078$ & $-0,005459$ & $-0,005459$ \\
0 & 0,032178 & 0,029100 & $-0,035594$ & $-0,041053$ \\
\hline 1 & 0,000199 & 0,029299 & $-0,002815$ & $-0,043868$ \\
2 & 0,002459 & 0,031758 & 0,001651 & $-0,042217$ \\
\hline 3 & $-0,002793$ & 0,028966 & 0,005530 & $-0,036687$ \\
4 & 0,000620 & 0,029586 & 0,002359 & $-0,034328$ \\
\hline 5 & $-0,001418$ & 0,028168 & 0,000254 & $-0,034074$ \\
6 & 0,001633 & 0,029802 & 0,001543 & $-0,032531$ \\
\hline 7 & $-0,007252$ & 0,022550 & $-0,000363$ & $-0,032894$ \\
8 & $-0,004534$ & 0,018016 & 0,000670 & $-0,032224$ \\
\hline 9 & 0,000365 & 0,018381 & $-0,003169$ & $-0,035393$ \\
10 & 0,002704 & 0,021085 & $-0,002288$ & $-0,037681$ \\
\hline
\end{tabular}

a) Análise da $\mathrm{H}_{1}$ : o mercado brasileiro no período estudado é eficiente após eventos favoráveis.

Quando um evento favorável ocorre tem-se um RAA de aproximadamente $+0,0291$, tendo seu maior nível no segundo dia subseqüente, e gradativamente vai havendo uma queda, conforme Figura 2, em que a linha contínua aponta toda a trajetória dos RAAs e a pontilhada une o primeiro dia, 0 de maior nível e o último.

$\mathrm{Na} \mathrm{HME} \mathrm{segundo} \mathrm{Fama} \mathrm{(1970)} \mathrm{os} \mathrm{preços} \mathrm{dos} \mathrm{ativos} \mathrm{financeiros} \mathrm{deveriam} \mathrm{reagir} \mathrm{de} \mathrm{forma}$ rápida e precisa à divulgação de qualquer notícia que afetar seus fundamentos de forma que não haja nem tendência ao exagero, nem a reversão dos preços após a divulgação, portanto, rejeita-se $\mathrm{H}_{1}$ visto que os resultados levam a entender que há uma sobrevalorização da informação, o que caracteriza aqui a Hipótese de Overreaction $(\mathrm{OH})$. Analisando a linha pontilhada do gráfico e comparando com a Figura 1 observa-se que ela se assemelha a $\mathrm{OH}$ para eventos favoráveis. 


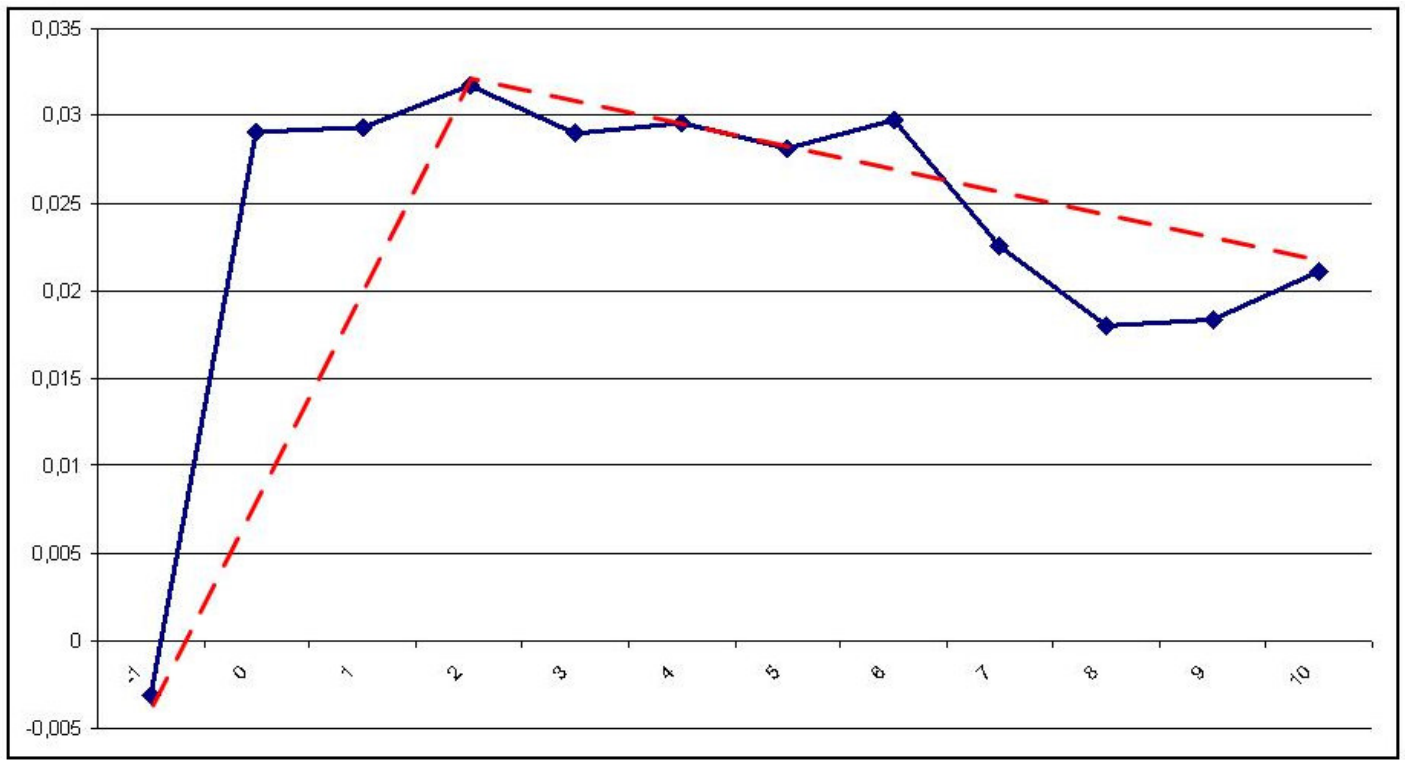

Figura 2: Choques Positivos.

b) Análise da H2: o mercado brasileiro no período estudado é eficiente após eventos desfavoráveis.

Investigando os choques negativos, quando houve um evento desfavorável, o RAA foi de 0,041053 , alcançando seu nível mais baixo no dia subseqüente e ele foi aumentando gradativamente, tendendo ao equilíbrio, conforme Figura 3 na qual a linha contínua demonstra toda a trajetória dos RAAs e a pontilhada une o primeiro dia, o de menor nível e o último.



Figura 3: Choques Negativos.

Como já foi referenciado na análise da hipótese $H_{1}$ na HME não pode haver nem tendência a exagero nem a reversão nos preços depois de uma divulgação, portanto, rejeita-se a hipótese $\mathrm{H}_{2}$ uma 
vez que, aqui, há uma tendência ao exagero, já que os preços caem acentuadamente e, com o passar do tempo, eles vão aumentando, caracterizando a Hipótese do Overreaction $(\mathrm{OH})$ e também a Uncertain Information Hypothesis $(\mathrm{UIH})$ já que, nessa hipótese de mercado, depois dos anúncios negativos as volatilidades são maiores do que nos positivos. Isto é uma conseqüência racional dos agentes que possuem aversão ao risco. Analisando a linha pontilhada do gráfico e comparando com a Figura 1 verifica-se que ela se assemelha a $\mathrm{OH}$ e a UIH para eventos desfavoráveis.

\section{CONCLUSÃO}

Neste estudo encontram-se evidências de que, no período analisado, o mercado brasileiro apresenta indícios de overreaction tanto para eventos positivos quanto para os negativos. A resposta de tendência ao exagero levou à rejeição das duas hipóteses levantadas de que o mercado brasileiro é eficiente para eventos favoráveis ou desfavoráveis.

Uma explanação possível é a de que o mercado brasileiro é relativamente pequeno quando medido pelo número de títulos listados e quantidade de investidores. Geralmente tais bolsas de tamanho reduzido são dominadas por pequeno número de investidores que respondem melhor às flutuações de mercado no longo prazo do que no curto prazo.

Essa tendência ao exagero ou sobrevalorização da informação também pode ser justificada pelo fato de os investidores não diversificarem tanto suas carteiras como sugere a teoria de finanças, por acabarem seguindo "gurus" financeiros, efetuarem uma excessiva negociação de ações, fazerem freqüentes trocas em suas carteiras, procurarem padrões nos preços das ações, terem a idéia de vender na alta e comprar na baixa (como diz o jargão do mercado financeiro).

Outro fator que poderia justificar esse fenômeno é a ação dos investidores não informados (noise traiders) isto é, investidores que agem motivados apenas por boatos e não em resposta a fatos concretos.

Com base na teoria supõe-se que os indivíduos se comportam de maneira racional. Conseqüentemente, os preços são ajustados para refletir toda informação disponível.

A chegada de anúncios inesperados conduz o investidor a chocar com o paradigma da eficiência.

Os distúrbios de mercado induzem os agentes financeiros a sobrevalorizar a informação após eventos positivos e negativos. De forma adicional, os mercados menores devem ser mais suscetíveis a essa sorte do comportamento irracional.

Tanto para eventos positivos quanto para os negativos não se constatou a eficiência de mercado.

No presente estudo foram analisados dez dias após os choques, e com base nessa análise conclui-se que, no período estudado, o mercado brasileiro revela evidências que suportam a Hipótese de Overreaction após eventos favoráveis e também após eventos desfavoráveis, sendo que esses últimos também se encaixam na Uncertain Information Hypothesis.

Para futuras pesquisas fica a sugestão de comparar o mercado brasileiro com o de outros países da América Latina ou com o de outros países emergentes seguindo a mesma metodologia utilizada neste estudo. 


\section{REFERÊNCIAS}

BROWN, K. C.; W. V. HARLOW; S. M. TINIÇ. Risk Aversion, Uncertain Information, and Market Efficiency. Journal of Financial Economics, V. 22, pp. 355-85, 1988.

FAMA, E. F. Efficient Capital Markets: a Review of Theory and Empirical Work. Journal of Finance, 25, 383-417, 1970.

LAMOUNIER, W. M.; NOGUEIRA, Else Monteiro. Estudo de Eventos: Procedimentos e Estudos Empíricos. In: II Seminário de Gestão de Negócios, 2005, Curitiba. II Seminário de Gestão de Negócios - Um Enfoque Acadêmico da Realidade Empresarial, 2005.

MEDEIROS, Otavio de. R. Reaction of the Brazilian Market to Positive and Negative Shocks. In: Global Finance Conference, 2006, Rio de Janeiro. Global Finance Conference Proceedings. Rio de Janeiro, 2006.

\section{SHACHMUROVE, Y. The Behavior of Secondary European Stock Markets to Positive and}

Negative Shocks. International Journal of Business, 7(2), 2002.

SOARES, João; SERRA, Ana Paula. Overreaction and Underreaction: Evidência do Mercado

Accionista Português. Cadernos do Mercado de Valores Mobiliários, V. 22, pp 55-84

\section{EMPIRICAL TEST OF THE EFFICIENCY OF THE BRAZILIAN MARKET IN THE OCCURRENCE OF EVENTS FAVORABLE AND UNFAVORABLE}

The study has the purpose of analyzing the behavior of the Brazilian stock market in order to verify the existence of market efficiency immediately after the occurrence of favorable and unfavorable events (shocks). To achieve this purpose, an event study is performed in which the return on the Brazilian stock market index (Ibovespa) is regressed against the return on the Dow Jones stock market index, which represents the New York Stock Exchange, adopted as a proxy for the world stock market index. Regression residuals appearing as outliers above $+2.5 \%$ or below $-2.5 \%$ were adopted to determine positive and negative events, respectively. Cumulative Abnormal Returns were computed and tested for a period of 10 days after the events. The empirical results led to the conclusion that market efficiency is not observed both after positive and negative shocks, but an overreaction behavior is observed instead.

Key words: economic shocks. Market efficiency. Overreaction. Uncertain information hypothesis. Underreaction. Event study.

Data de Submissão: fevereiro de 2007 Data de Aceite: junho de 2007 